Involutions in groups of finite Morley rank degenerate type

Borovik, Alexandre and Burdges, Jeffrey and Cherlin, Gregory 2007

MIMS EPrint: 2007.177

Manchester Institute for Mathematical Sciences

School of Mathematics

The University of Manchester

\footnotetext{
Reports available from: http://eprints.maths.manchester.ac.uk/

And by contacting: The MIMS Secretary

School of Mathematics

The University of Manchester

Manchester, M13 9PL, UK
} 


\title{
Involutions in groups of finite Morley rank of degenerate type
}

\author{
Alexandre Borovik, Jeffrey Burdges and Gregory Cherlin
}

\begin{abstract}
In a connected group of finite Morley rank, if the Sylow 2-subgroups are finite then they are trivial. The proof involves a combination of modeltheoretic ideas with a device originating in black box group theory.
\end{abstract}

Mathematics Subject Classification (2000). 03C60, 20 G99.

\section{Introduction}

Modern model theory can be viewed as a subject obsessed with notions of dimension, with the key examples furnished by linear dimension on the one hand, and the dimension of an algebraic variety (or, from another point of view, transcendence degree) on the other. There are several rigorous, and not always equivalent, notions of abstract dimension in use. For historical reasons the one we use is generally referred to as Morley rank. In the applications of model theory, it is important that this dimension may be ordinal valued, but the case of finite dimension continues to stand out. For example, in the model-theoretic approach to the Manin kernel in an abelian variety, one enriches the underlying algebraically closed field with a differential field structure, at which point the abelian variety becomes infinitedimensional, but the Manin kernel itself is finite-dimensional, which accounts for a certain number of its fundamental properties.

For some time it was hoped that one would be able to classify the "onedimensional" objects arising in model theory explicitly and in complete generality, a hope which was dashed by a construction of Hrushovski. But in diophantine applications, even after leaving the algebraic category, one has in addition to the dimension notion a topology reminiscent of the Zariski topology, and some very strong axioms, given in [HZ96]. In this case one gets the desired algebraicity result, in nondegenerate cases, with substantial diophantine applications (cf. [Bo98, Sc01]). 
The work reported here concerns the Algebraicity Conjecture (Cherlin/Zilber) which states that a simple group of finite Morley rank should be algebraic. This occupies a middle position between the known results used in diophantine applications (where the focus in any case is on abelian groups) and the more ambitious conjectures which have been refuted. There is no assumption of a topological nature, and the axioms are only those which occur in general model theory, but considerably refined by results holding in the specific context of groups, where the group action introduces a degree of uniformity into the picture.

This algebraicity conjecture also occupies a kind of middle position between algebraic group theory and finite group theory. The identification of the simple algebraic groups as Chevalley groups can be carried out with relentless efficiency by examining maximal tori and their actions on unipotent subgroups, thereby quickly revealing the associated root system and giving the structure of the Weyl group. The classification of simple finite groups is similar in outcome: setting aside the alternating groups and a sackful of sporadic groups, one has some sort of twisted Chevalley group, which can be identified by determining the associated building, though the process is so intricate that by the time the building is actually visible the whole group is equally visible. The question arises whether either of these two approaches offers anything for our more general problem. In particular cases, both do, and they can even be combined. But this requires a certain supply of elements of order two to implement.

Some time ago a project was launched to apply the techniques of finite simple group theory in combination with relevant notions of algebraic group theory, toward an analysis of hypothetical nonalgebraic simple groups of finite Morley rank containing involutions, which aims at pinning down the critical (minimal) configurations with as much precision as possible. An early sketch of the possibilities is found in BN94. In some cases the hypothesis of minimality is superfluous: for example, if the group contains a nontrivial unipotent 2-subgroup in an appropriate sense $\mathrm{ABC}$. Another such case is the case in point in the present article. We will prove the following.

Theorem 1. Let $G$ be a connected group of finite Morley rank whose Sylow 2subgroup is finite. Then $G$ contains no involutions - the Sylow 2-subgroup is trivial.

As a simple group will be either finite or connected, this result tells us in the simple case that the only exceptions are the finite simple groups. The following version is a little sharper.

Theorem 2. Let $G$ be a connected group of finite Morley rank, and a $\in G$ an involution. Then the Sylow 2-subgroup of $C(a)$ is infinite.

This casts considerable light on the general program of determining the possible 2-Sylow structure in a hypothetical counterexample to the Cherlin/Zilber Algebraicity Conjecture, according to which simple groups of finite Morley rank should be Chevalley groups. 
Groups of finite Morley rank are abstract groups equipped with a notion of dimension which assigns to every definable set $X$ a dimension, called Morley rank and denoted $\operatorname{rk}(X)$, satisfying well known and fairly rudimentary axioms given for example in [BN94, Po01]. Examples are furnished by algebraic groups over algebraically closed fields, with $\operatorname{rk}(X)$ equal to the dimension of the Zariski closure of $X$. The Algebraicity Conjecture amounts to the assertion that simple algebraic groups can be characterized, as abstract groups, by the presence of a dimension. A striking feature of this conjecture is the complete absence of any topological assumptions; as far as this circle of ideas is concerned, it is the main conjecture which survives without imposing topological conditions.

As the category of groups of finite Morley rank is closed under finite direct products, it is easy to fabricate nonalgebraic examples, but to construct "seriously" nonalgebraic examples is a challenge, which so far has been best met by Baudisch in Ba96] with a nilpotent example.

To put the ongoing work in context, we need to present an overall framework for the analysis, as well as the current status of the program. One first defines Sylow 2-subgroups in the natural way, and one also considers the connected components of Sylow 2-subgroups, which are called Sylow ${ }^{\circ} 2$-subgroups. The structure of these Sylow ${ }^{\circ} 2$-subgroups is as follows, in the context of groups of finite Morley rank:

$$
S=U * T, \quad U \text { 2-unipotent, } T \text { a 2-torus. }
$$

That is, $S$ is the central product of groups $U$ and $T$ where $U$ is definable and connected, and of bounded exponent, while $T$ is a divisible abelian 2-group, not in general definable. This simple fact provides a framework for further analysis.

It is known that necessarily $S=U$ or $S=T$ when the ambient group $G$ is simple, the other factor being trivial [ABC]. Furthermore, when $U$ is nontrivial the group $G$ is in fact algebraic. These are substantial results relying on an analysis which would be considered long by most standards, though unbelievably rapid by the standards of finite simple group theory. So attention focusses on the case $S=T$, which in fact is two cases:

$$
S=T>1 \text { (odd type); } S=T=1 \text { (degenerate type). }
$$

We note that another formulation of the degeneracy condition is that the full Sylow 2-subgroup of the ambient group $G$ is finite, and that is the condition we have adopted in formulating our main result above.

It is in odd type that the theory still relies on the assumption that the ambient simple group is a $K^{*}$-group; that is, all its proper definable simple sections are algebraic. Given that, if the ambient simple group is nonalgebraic then the group $T$ satisfies severe restrictions: it is known that its Prüfer rank (which is the same as the 2-rank $m_{2}(T)$ ) is at most two, which is a condition analogous to Lie rank at most two in the algebraic case. A good deal more is known about this case, and a good deal remains to be done here.

Throughout, the case of degenerate type has occupied a peculiar position. It has certainly not been ignored, but on the other hand nothing approaching a 
systematic plan or point of view has ever been found for dealing with this case. The elimination of involutions from these groups has been arrived at unexpectedly, and from an unusual direction: techniques used in the computational theory of so-called black box groups provide a key ingredient in the proof, and the remaining ingredients are purely model-theoretic. What we borrow is taken from one particular chapter in the theory which owes little to the conventional analysis of finite simple groups, other than a preoccupation with centralizers of involutions. The sort of problem we are dealing with here (which is related to the $Z^{*}$-theorem in finite group theory) would normally be approached using character-theoretic methods and transfer arguments, neither of which have analogs in our setting. The black box methods become very global and direct in our setting, having to do with the ranks of fibers of appropriate covariant maps (first in a rudimentary way in $\S 3$, then more precisely in $\S 5)$.

Tuna Altinel has pointed out that with Theorem 1 in hand, our subject reaches a level of maturity sufficient to dispose of two "chestnuts" from the general theory of groups of finite Morley rank by reduction to the simple case and application of what amounts to a tripartite theory (degenerate type, odd type, and even type - the last being the case $S=U>1$ ). Here the fact that odd type groups are always studied in an inductive setting could be an obstacle, but as it happens a certain amount of information of a noninductive character has also been obtained recently [Ch05]. The two results in question are the following.

Proposition 1.1. Let $G$ be a connected and nontrivial group of finite Morley rank. Then the centralizer of any element of $G$ is infinite.

Proposition 1.2. Let $G$ be a connected group of finite Morley rank containing a definable generic subset whose elements are of order $2^{k}$ for some fixed $k$. Then $G$ has exponent $2^{k}$.

Both of these problems have been notoriously open. The point of the second one, stressed by Poizat, is that the corresponding result for algebraic groups is trivial, in view of the presence of a suitable topology [Po90, Po01, p. 145].

One can combine these two results as follows.

Proposition 1.3. Let $G$ be a connected group of finite Morley rank containing a definable generic subset whose elements are of order $n$ for some fixed $n$. Then the Sylow 2-subgroup $S$ of $G$ is unipotent, $G=S * C_{G}(S)$ is a central product, and $G / S$ is a group without involutions whose elements are generically of order $n_{0}$, where $n_{0}$ is the odd part of $n$.

Thus for all practical purposes the study of the "generic equation" $x^{n}=1$ reduces to the case of $n$ odd. The analysis in that case is complicated by the possible existence of simple $p$-groups of finite Morley rank.

While Proposition 1.3 is expressed as a strong form of Proposition 1.2, it also includes Proposition [1.1 if $a \in G$ has a finite centralizer, then $a$ has finite order and its conjugacy class $a^{G}$ is generic in $G$. Then applying Proposition 1.3, 
evidently $G>S$ and thus $\bar{G}=G / S$ is a group without involutions in which the conjugacy class of the image $\bar{a}$ of $a$ is still generic. But the same applies to $\bar{a}^{-1}$ and thus $\bar{a}, \bar{a}^{-1}$ are conjugate, and this produces involutions in $G / S$, a contradiction.

We remark that these last results (and Theorems 3 , 4 following) rely on a considerable body of material, not all of it fully published at this time, and the reader may prefer to consider them as conditional - specifically, conditional on the classification of groups of even type $\mathrm{ABC}$.

We will generalize Theorem 1 as follows.

Theorem 3. Let $G$ be a connected group of finite Morley rank whose Sylow psubgroups are finite. Then $G$ contains no elements of order $p$.

Although the proof for odd $p$ involves a reduction to the statement for $p=2$, it will be seen that parts of the two proofs can be done uniformly.

Then we can continue on to the parallel to Theorem 2 .

Theorem 4. Let $G$ be a connected group of finite Morley rank, and $a \in G$ a $p$ element. Then the Sylow p-subgroup of $C(a)$ is infinite.

Returning to the case $p=2$, we will also show the following.

Theorem 5. Let $V$ be a 4-group acting definably on a connected group $H$ of finite Morley rank and degenerate type. Then

$$
H=\left\langle C_{H}^{\circ}(v): v \in V^{\times}\right\rangle .
$$

In fact one first applies Theorem 1 to reduce to the involution-free case (and in that setting, connectivity is no longer needed). However, this more general form is the form one would actually want, and may actually be useful, eventually, in emancipating the odd type analysis from the $K^{*}$-hypothesis, a line of development which remains to be explored. In this line, the following is completely open.

Problem 1. Can one show that a simple group of finite Morley rank and degenerate type has no nontrivial involutory automorphism?

Evidently the hypothesis of simplicity is necessary, but is not easy to bring to bear on the problem.

Finally, we should mention an outstanding open question of a general nature concerning groups of finite Morley rank.

Conjecture 1 (Genericity Conjecture). Let $G$ be a connected group of finite Morley rank. Then the union of the connected definable nilpotent subgroups of $G$ contains a definable generic subset of $G$.

This conjecture reflects quite well a useful property of connected algebraic groups. Its truth would greatly simplify the proof of our main result. Conversely, some of our methods might contribute to the general analysis of this problem. Jaligot has shown [Ja06] that statements closely related to the Genericity Conjecture have strong implications for the general structure theory of groups of finite Morley rank, and mesh well with the existing Carter subgroup theory. 
Notation follows [BN94]. We mention the notation $d(g)$ for the definable hull of $g$ in the group-theoretic sense: this is the smallest definable subgroup containing $g$, and coincides with $d(\langle g\rangle)$. See [BN94, §5 et passim] for this.

We thank Altinel for a number of useful discussions, not confined to the points already mentioned above.

\section{Approximating $d(g)$}

The present section has a purely technical character. We will make extensive use of the function $d(g)$ which assigns to an element $g$ the smallest definable subgroup containing it, which we think of as the definable group "generated by" $g$. Unfortunately the function $d$ is not in general definable (in an algebraic geometric context the parallel remark would be that the family $\{d(g): g \in G\}$ is not an algebraic family). Indeed, for $g$ of finite order the group $d(g)$ is the finite cyclic group generated by $g$, which is typically of unbounded order, and hence cannot be given in a uniformly definable way.

We will replace $d$ by a definable approximation $\hat{d}$ with essentially the same properties from a practical point of view. There is no "canonical" approximation, but we will record all the properties of $\hat{d}$ used in the present paper, which we think provides a robust approximation to the function $d$.

A word on the terminology used in the next lemma is in order. We are interested in $p$-torsion (elements of order $p^{n}$ ) for all primes $p$, with special interest in the case $p=2$. We will work generally with the hypothesis that Sylow $p$-subgroups have finite exponent. There are two distinct notions of Sylow $p$-subgroup, both reasonably natural, in this context: either a maximal $p$-subgroup, as usual, or else a maximal locally nilpotent $p$-subgroup (local nilpotence can be replaced here by anything similar-local finiteness or solvability for example).

With either definition, there is at present no Sylow theory for $p$ odd, except in the framework of solvable groups. Note however that the following conditions are equivalent.

(1) Every abelian $p$-subgroup of $G$ has finite exponent.

(2) Every Sylow $p$-subgroup of $G$ has finite exponent.

(3) The $p$-torsion of $G$ has bounded exponent.

Since these are successively stronger conditions, it suffices to show that the first implies the last. If the $p$-torsion of $G$ has unbounded exponent, then in a saturated elementary extension $G^{*}$ of $G$ there will be an infinite quasicyclic $p$ subgroup $A$. Then $d(A)$ will be a definable abelian $p$-divisible group containing an element of order $p$. The existence of such a subgroup passes from $G^{*}$ to $G$ and contradicts (1).

Since all of these conditions are equivalent, we may express them as follows: " $G$ has Sylow $p$-subgroups of bounded exponent." The reader may possibly prefer to keep clause (3) in mind. 
The practical effect of this condition is the following, which may be surprising if one thinks in terms of "cyclic" subgroups; the correct intuition is furnished by Zariski closures of cyclic subgroups.

Lemma 2.1. Let $G$ be a group of finite Morley rank, $p$ a prime. Suppose that $G$ has Sylow p-subgroups of bounded exponent, and let $p^{n}$ be a bound on the exponent. Then for any element $g \in G$, the following hold.

- $d\left(g^{p^{n}}\right)$ is uniquely p-divisible.

- $d(g)$ has a cyclic Sylow p-subgroup $S$.

- $d(g)=d\left(g^{p^{n}}\right) \times S$.

Proof. Let $A=d(g)$, a definable abelian group. One has $A=A_{0} \oplus S$ with $A$ $p$-divisible and $S$ a $p$-group of finite exponent. By our hypothesis, $A_{0}$ has no $p$ torsion and thus $S$ is the Sylow $p$-subgroup of $A$. We can write $g=a s$ with $a \in A_{0}$, $s \in S$ and then $g \in A_{0} \times\langle s\rangle$, so $d(g)=A_{0} \times\langle s\rangle$. All that needs to be checked at this point is that $A_{0}=d\left(g^{p^{n}}\right)$.

We have $g^{p^{n}}=a^{p^{n}}$. Since every definable subgroup of $A_{0}$ is $p$-torsion free and thus uniquely $p$-divisible, it follows that $a \in d\left(g^{p^{n}}\right)$ and thus $g \in d\left(g^{p^{n}}\right) \times S$. Hence $d(g)=d\left(g^{p^{n}}\right) \times S$ and $d\left(g^{p^{n}}\right)=A_{0}$.

The picture provided by the foregoing lemma should be borne in mind throughout. Note that tori behave quite differently; their generic elements are dense, and thus the Sylow $p$-subgroups of $d(g)$ in general can be very far from cyclic.

Lemma 2.2. Let $p$ be a prime, and let $G$ be a group of finite Morley rank with Sylow p-subgroups of bounded exponent. Then there is a definable function $\hat{d}(a)$, from elements of $G$ to definable subgroups of $G$, with the following properties:

(1) $d(a) \leq \hat{d}(a)$.

(2) If $d(a)=d(b)$, then $\hat{d}(a)=\hat{d}(b)$.

(3) For $g \in G$, we have $\hat{d}\left(a^{g}\right)=\hat{d}(a)^{g}$.

(4) $\hat{d}(a)$ is abelian.

(5) The groups $d(a)$ and $\hat{d}(a)$ have the same Sylow p-subgroup.

(6) If $x \in G$ conjugates a to its inverse, then $x$ normalizes $\hat{d}(a)$ and acts on it by inversion.

Proof. Consider the following two functions:

- $d_{1}(a)=Z(C(a))$.

- $d_{2}(a)=d_{1}(a)^{q}\langle a\rangle$ where $q$ is a bound on the order of the $p$-torsion in $G$.

One sees easily that $d_{1}$ is definable and satisfies our first four conditions (cf. [BN94, $\S 5.1])$. As $\left[d_{2}(a): d_{1}(a)^{q}\right] \leq q$ it follows easily that $d_{2}$ is also a definable function, and it also satisfies condition (1) and inherits conditions (2)-(4) from $d_{1}$.

Furthermore, $d_{2}(a)$ also satisfies the fifth condition, since $d_{1}(a)$ is abelian and $d_{1}(a)^{q}$ is $q$-torsion free.

Now to achieve the final point, let $d_{3}(a)$ be the subgroup of $d_{2}(a)$ consisting of elements inverted by every element that inverts $a$. 
The following is in a similar vein.

Lemma 2.3. Let $G$ be a group of finite Morley rank, and $p$ a prime, with Sylow $p$-subgroups of $G$ of bounded exponent. Let $H$ be a definable subgroup of $G$, and $a \in N(H)$ of order $p$ modulo $H$. Then $d(a) \cap a H$ contains a p-element.

Proof. Letting $q$ be the order of a Sylow $p$-subgroup $S$ of $d(a)$, we have $d(a)=$ $d\left(a^{q}\right) \times S$ and thus $a H=s H$ for some $s \in S$.

In the case that interests us, the group $H$ will contain no elements of order $p$, and then the $p$-element in $a H \cap d(a)$ will be unique and of order $p$.

\section{Minimization}

Lemma 3.2 below will describe the situation arising from consideration of a hypothetical minimal counterexample to Theorem 1 or 3 .

Definition 3.1. Let $G$ be a group of finite Morley rank and $p$ a prime. We say that $G$ is $p$-degenerate if $G$ contains no infinite abelian $p$-subgroup.

Observe that $G$ is 2-degenerate if and only if $G$ is of degenerate type.

Lemma 3.2. Let $G$ be a connected p-degenerate group of finite Morley rank with nontrivial p-torsion, and of minimal Morley rank among all such groups. Then $\bar{G}=G / Z(G)$ is simple, and contains nontrivial $p$-torsion, while no proper definable connected subgroup of $\bar{G}$ contains nontrivial p-torsion.

Proof. By our minimality hypothesis no proper connected subgroup of $G$ contains $p$-torsion. If $H<G$ is a nontrivial definable connected normal subgroup, then passing to $G / H$ we contradict the minimality of $G$. So $Z(G)$ is finite and $G / Z(G)$ is simple. It suffices to show that $G / Z(G)$ contains nontrivial $p$-torsion.

Supposing the contrary, after passing to a quotient of $G$ we may suppose that $Z(G)$ is a $p$-group. We now introduce a function

$$
\eta: G \rightarrow Z(G)
$$

which though not a homomorphism will be covariant with respect to the action of $Z(G)$. This is defined as follows.

For $g \in G$, we consider the subgroup $\hat{d}(g)$, which splits as $\hat{d}(g)^{q} \times S_{g}$, with $q$ the exponent of $Z(G)$ and $S_{g} \leq Z(G)$ the Sylow $p$-subgroup of $\hat{d}(g)$ (or of $d(g)$ ). So the projection $\pi_{2}: \hat{d}(g) \rightarrow S_{g}$ is well-defined, and we may set $\eta(g)=\pi_{2}(g) \in Z(G)$.

The desired covariance property is the following:

$$
\eta(z g)=z \eta(g) \quad \text { for } z \in Z(G), g \in G .
$$

Writing $g=g_{0} s$ with $g_{0} \in d(g)^{q}$ and $s \in S_{g}$, we have $g_{0}^{q} \in d(z g)$ and as $d\left(g_{0}\right)$ is uniquely $p$-divisible we have $g_{0} \in d(z g)$. But $z g=g_{0} z s$ and hence $z s \in d(z g) \leq$ $\hat{d}(z g)$ as well, and our claim follows. 
Now in view of the covariance of the map $\eta$, its fibers have constant rank. Thus $G$ is partitioned by the fibers of $\eta$ into finitely many sets of equal rank, and as $G$ is connected this yields a contradiction.

\section{Genericity}

In the present section we suppose the following.

$G$ is a $p$-degenerate simple group of finite Morley rank, $p$ is a prime, $G$ contains nontrivial $p$-torsion, and no proper definable connected subgroup of $G$ contains nontrivial $p$-torsion.

Let $q$ be a bound on the exponent of the $p$-torsion in $G$.

We will show that the generic elements of $G$ lie outside every proper connected subgroup of $G$, and we will pin down their location with sufficient precision to give useful structural information. In particular we will show that the Sylow $p$-subgroup of $G$ has exponent $p$, and that any two elements of order $p$ are conjugate.

We begin with a rank computation similar to that of [CJ04, 3.3], but slightly more general.

Lemma 4.1. Let $G$ be a group of finite Morley rank, $H$ a definable subgroup of $G$, and $X \subseteq G$ a definable set such that

$$
\operatorname{rk}\left(X \backslash \bigcup_{g \notin H} X^{g}\right) \geq \operatorname{rk}(H) .
$$

Then $\operatorname{rk}\left(\bigcup X^{G}\right)=\operatorname{rk}(G)$.

Proof. Let $Y=X \backslash \bigcup_{g \notin H} X^{g}$. Let $\hat{Y}=\bigcup Y^{H}$. Then for $h \in H$ and $g \notin H$ we have $Y^{h} \cap Y^{g}=\left(Y \cap Y^{g h^{-1}}\right)^{h}=\emptyset$, and thus $\hat{Y} \cap \hat{Y}^{g}=\emptyset$. Furthermore $\operatorname{rk}(\hat{Y}) \geq \operatorname{rk}(Y) \geq \operatorname{rk}(H)$.

Now $H$ is the setwise stabilizer of $\hat{Y}$ in $G$ and so the family $\mathcal{F}=\left\{\hat{Y}^{g}: g \in G\right\}$ has $\operatorname{rank} \operatorname{rk}(G)-\operatorname{rk}(H)$. As distinct elements of $\mathcal{F}$ are disjoint, we have

$$
\operatorname{rk}(\bigcup \mathcal{F})=\operatorname{rk}(\hat{Y})+\operatorname{rk}(\mathcal{F}) \geq \operatorname{rk}(G) .
$$

Thus $\operatorname{rk}\left(\bigcup \hat{Y}^{G}\right)=\operatorname{rk}(G)$, and the same applies to $\bigcup X^{G}$.

In practice the group $H$ is the generic stabilizer of $X$ in the following sense.

Definition 4.2. Let $G$ be a group of finite Morley rank and $X \subseteq G$ definable. The (generic) stabilizer $G[X]$ is the subgroup

$$
\left\{g \in G: \operatorname{rk}\left(X \triangle X^{g}\right)<\operatorname{rk}(X)\right\} .
$$


The way we obtain the hypotheses of our lemma in practice is by showing the following, in each instance:

$$
\operatorname{rk}\left(X \backslash \bigcup_{g \notin G[X]} X^{g}\right)=\operatorname{rk}(X) \geq \operatorname{rk}(G[X]) .
$$

We may then take $H=G[X]$.

Sometimes $X$ is even disjoint from its conjugates $X^{g}(g \notin G[X])$ but more often in applications this strong condition fails but our weaker condition holds.

Lemma 4.3. Suppose $a$ is a p-element of $G$. Then the set $\bigcup\left(a C^{\circ}(a)\right)^{G}$ is generic in $G$.

Proof. We remark that $C^{\circ}(a)$ contains no $p$-torsion, by our hypothesis.

It will suffice to show that $a C^{\circ}(a)$ is disjoint from its conjugates $\left(a C^{\circ}(a)\right)^{g}$ for $g \notin C(a)$, as we may then apply Lemma 4.1.

So suppose we have an element $x \in\left(a C^{\circ}(a)\right) \cap\left(a C^{\circ}(a)\right)^{g}$, with $g \in G$. Then $d(x)=A \oplus Z$ with $A p$-divisible and $Z$ a cyclic $p$-group, in fact $Z=\langle a\rangle$. Accordingly $C^{\circ}(Z)=C^{\circ}(a)$ and $a$ is the only $p$-element in the coset $x C^{\circ}(Z)$. Similarly, since $x \in\left(a C^{\circ}(a)\right)^{g}$, we find that $a^{g}$ is the only $p$-element in $x C^{\circ}(Z)$.

Thus $a=a^{g}$ and $g \in C(a)$.

Now we can pass to structural consequences. We emphasize that the clause $(\dagger)$ has been assumed throughout.

Lemma 4.4. All nontrivial p-elements a of $G$ are $G$-conjugate and of order $p$, and if $p=2$ then the Sylow 2-subgroup is elementary abelian.

Proof. For $a, b$ nontrivial $p$-elements of $G$, the sets $\bigcup\left(a C^{\circ}(a)\right)^{G}$ and $\bigcup\left(b C^{\circ}(b)\right)^{G}$ are generic in $G$ and so we may suppose after conjugating that $a C^{\circ}(a) \cap b C^{\circ}(b) \neq \emptyset$. Now if $x \in a C^{\circ}(a) \cap b C^{\circ}(b)$ then as in the proof of the previous lemma, $a=b$.

So the $p$-elements are conjugate, and hence are all of order $p$. In particular when $p=2$ the Sylow 2-subgroup is elementary abelian.

This is about as far as the genericity arguments take us, and it is time to focus on the case $p=2$ and black box methods.

\section{Black box methods}

Now we specialize condition $(\dagger)$ to the case $p=2$. Our hypotheses will therefore be as follows.

$G$ is a degenerate type simple group of finite Morley rank, $G$ contains involutions, and no proper definable connected subgroup of $G$ contains involutions.

For the moment we need not rely on the results of the previous section. Rather we introduce a crucial case division, dispose of one case using so-called black box 
group-theoretic methods, and then return to the other case using the information from the last section.

We turn to the case division. Fix a conjugacy class of involutions $\mathcal{C}$, and note that as this set can be identified definably with $G / C(i)$ for any fixed $i \in \mathcal{C}$, it has Morley degree one. Thus the notion of "generic element" or pair of elements in $\mathcal{C}$ is robust. The first case we will treat is the following one:

For generic and independent $i, j \in \mathcal{C}$ the group $d(i j)$ contains no involution.

(Case I)

The following two facts are elementary but important, and are used in combination.

Lemma 5.1. Let $G$ be a group of finite Morley rank and a an element of $G$. Suppose that the Sylow 2-subgroups of $G$ have bounded exponent. Then the following conditions on the group $d(a)$ are equivalent:

(1) $d(a)$ contains no involutions.

(2) $d($ a) is 2-divisible.

(3) $d(a)$ is uniquely 2-divisible.

On the other hand, if $d(a)$ does contain an involution, then that involution is unique.

Lemma 5.2. Let $G$ be a group of finite Morley rank. Suppose that the Sylow 2subgroups of $G$ have bounded exponent. Let $i, j$ be involutions of $G$, and let $a=i j$. Then $d(i, j)=d(a) \rtimes\langle i\rangle$, where $i$ acts by inversion on $d(a)$. Furthermore, $i$ and $j$ are conjugate under the action of $d(a)$ if and only if $d(a)$ contains no involution.

This is well-known in the finite case and the infinite case is much the same. The only noteworthy point is the fact that in the absence of 2-torsion the group is 2divisible, a point already used repeatedly but relying essentially on the definability of the group in question.

This leads to consideration of the following partial functions from the group $G$ to $C(i)$, for any fixed involution $i$, under the hypothesis that the Sylow 2-subgroups of $G$ have bounded exponent:

(1) $\zeta_{0}(g)$ is the unique involution in $d\left(i \cdot i^{g}\right)$, if $d\left(i \cdot i^{g}\right)$ contains an involution;

(2) $\zeta_{1}(g)$ is the unique element in $g d\left(i \cdot i^{g}\right) \cap C(i)$, if $d\left(i \cdot i^{g}\right)$ contains no involution.

Indeed, under our hypothesis we have just seen that $\zeta_{0}$ is well-defined on its domain. On the other hand, if $d\left(i \cdot i^{g}\right)$ contains no involution then there is an element $x \in d\left(i \cdot i^{g}\right)$ conjugating $i$ to $i^{g}$, so $g x^{-1}$ belongs to $C(i) \cap g d\left(i \cdot i^{g}\right)$. As far as uniqueness is concerned, if $g x, g y \in C(i) \cap g d\left(i \cdot i^{g}\right)$, then $x^{-1} y \in C(i) \cap d\left(i \cdot i^{g}\right)$ is both centralized and inverted by $i$, hence an involution or trivial, and as we have ruled out involutions we conclude $x=y$. One could also compute more directly that $x^{-1}=x^{i}=\left[i, x^{-1}\right] x=[i, g] x$ and thus $x^{2}=\left(i i^{g}\right)^{-1}$, so that $x$ is uniquely determined within $d\left(i \cdot i^{g}\right)$, symbolically $x=\sqrt{\left(i i^{g}\right)^{-1}}$, with the square 
root operation restricted to $d\left(i \cdot i^{g}\right)$, though this extra precision is useful mainly as a way of verifying the existence of $x$.

The functions $\zeta_{0}$ and $\zeta_{1}$ are definable, because we can replace $d$ by $\hat{d}$ everywhere in their definitions, and the Sylow 2-subgroups remain the same. The uniqueness argument also relies on the properties of $\hat{d}$ given at the outset, which mimic the properties of $d$.

Lemma 5.3. Case I does not occur.

Proof. We fix $i \in \mathcal{C}$ and consider the definable partial function $\zeta_{1}: G \rightarrow C(i)$ discussed above, which is defined on a generic subset of $G$, namely

$$
\zeta_{1}(g) \in C(i) \cap g d\left(i i^{g}\right) .
$$

It follows by inspection of the definition that we have the covariance property

$$
\zeta_{1}(c g)=c \zeta_{1}(g)
$$

for $g$ in the domain of $\zeta_{1}$ and $c \in C(i)$. This implies that the fibers of $\zeta_{1}$ are of constant rank, say $f$, and hence that any subset of $C(i)$ of rank $r$ lifts under $\zeta_{1}$ to a subset of $G$ of rank $r+f$. Now since $i \in C(i) \backslash C^{\circ}(i)$, the group $C(i)$ is disconnected and hence has disjoint subsets of full rank, and these lift under $\zeta_{1}$ to disjoint generic subsets of $G$, which contradicts the connectedness of $G$ [BN94, Theorem 5.12].

\section{Proof of Theorem 1}

We suppose toward a contradiction that $G$ is a connected group of degenerate type containing an involution. Applying Lemma 3.2 we may suppose condition $(\dagger)$ of $\S 5$ holds.

We fix a conjugacy class of involutions $\mathcal{C}$ in $G$, and in view of Lemma 5.3 we suppose that the following holds:

For generic and independent $i, j \in \mathcal{C}$ the group $d(i j)$ contains a unique involution.

(Case II)

Applying Lemma 4.4 we find that the Sylow 2-subgroups of $G$ are elementary abelian. This then yields the following.

Lemma 6.1. If $i, j$ are involutions and $k \in d(i j)$ is an involution, then $i$ and $j$ are not conjugate under the action of $C(k)$.

Proof. We show first that $i$ and $i k$ are not conjugate in $C(k)$. Suppose on the contrary $i^{u}=i k$ with $u \in C(k)$. Then $i^{u^{2}}=i$ and $u$ acts on the group $\langle i, k\rangle$ as a nontrivial automorphism of order two. It follows that $d(u)$ contains a 2-element with the same action, and as $G$ has abelian Sylow 2-subgroups this is impossible.

On the other hand, as in the case of ordinary dihedral groups one may see that the group $d(i, j)$ has two conjugacy classes of noncentral involutions, represented by $i$ and $j$, and in particular $j$ is conjugate to $i k$ under the action of $d(i j)$, and in 
particular under $C(k)$. If $i$ is conjugate to $j$ under $C(k)$ then $i$ is conjugate to $i k$ under $C(k)$ and we have a contradiction.

Now we may conclude the proof of Theorem 1 by a model-theoretic argument.

Fix a Sylow 2-subgroup $S$ of $G$ and consider a pair $i, j$ of involutions in $\mathcal{C}$ which are independent and generic over $S$, that is to say, with the elements of $S$ treated as constants. Define a subset $S_{i, j} \subseteq S \times S$ as follows:

$$
S_{i, j}=\{(s, t) \in S \times S:(i, k) \sim(s, t)\} .
$$

Here $k$ is the unique involution in $d(i j)$, and " $\sim$ " refers to conjugacy under the action of $G$. As $i$ and $k$ commute, the set $S_{i, j}$ is nonempty.

Now the pair $(i, j)$ and the pair $(j, i)$ have the same type over $S$, so $S_{i, j}=S_{j, i}$. As the involution $k$ is also the unique involution in $d(j i)$, this means that $(i, k)$ and $(j, k)$ are conjugate to the same pairs in $S \times S$, and hence to each other. But to conjugate $(i, k)$ to $(j, k)$ in $G$ means that $i$ is conjugated to $j$ in $C(k)$. This contradicts the preceding lemma and completes the proof of Theorem 1.

As mentioned in the introduction, we can also strengthen Theorem 1 as follows.

Theorem 2, Let $G$ be a connected group of finite Morley rank, and $i \in G$ an involution. Then the Sylow 2-subgroup of $C(i)$ is infinite.

Proof. Suppose toward a contradiction that $G$ and $i$ are a counterexample with $\operatorname{rk}(G)$ minimal. Then $i$ belongs to no proper definable connected subgroup of $G$.

By hypothesis $C^{\circ}(i)$ is of degenerate type and hence contains no involution. Hence for $c \in C^{\circ}(i), i$ is the unique involution in $d(c i)$. Now for $g \in G$, if $\left(i C^{\circ}(i)\right) \cap$ $\left(i C^{\circ}(i)\right)^{g} \neq \emptyset$ and $x$ lies in the intersection, then $i, i^{g} \in d(x)$ and thus $i=i^{g}$, and $g \in C(i)$. It follows that the distinct conjugates $\left(i C^{\circ}(i)\right)^{g}$ are disjoint for $g \in G$, and $i C^{\circ}(i)$ has stabilizer $C(i)$.

So by Lemma 4.1 we have $\operatorname{rk}\left(\bigcup\left[i C^{\circ}(i)\right]^{G}\right)=\operatorname{rk}(G)$. In particular, the generic element of $G$ lies outside every proper definable connected subgroup.

We claim that a Sylow ${ }^{\circ}$-subgroup $T$ of $G$ is a nontrivial 2-torus. It is nontrivial since $G$ is connected and contains an involution, and it is a 2 -torus since otherwise it would have a nontrivial unipotent factor $U$ which we may take to be normalized by $i$, and then $C_{U}(i)$ would be infinite. By [Ch05] the generic element of $G$ lies in a conjugate of $N^{\circ}(T)=C^{\circ}(T)$. So if $C^{\circ}(T)<G$ then the generic element lies in a proper definable connected subgroup, a possibility we have just ruled out. We are left with the possibility $C^{\circ}(T)=G$, and then of course $C_{T}(i)=T$. So in any case we reach a contradiction.

\section{Generic equations}

Now we take up Proposition 1.3. concerning generic equations of the form

$$
x^{n}=1
$$


In the statement of that proposition it was assumed that $n$ was the precise order of the generic element of $G$, but it will be convenient here to work with the slightly broader condition $(*)$, so that the order of a generic element is a fixed divisor of $n$. As we have noted, Altinel suggested that Theorem 1 supplies the missing piece of the puzzle to treat a substantial portion of this problem.

In the solvable case, with $n$ arbitrary, Jaber [Ja99] has shown by an argument that uses results of Bryant and Wagner that when such an equation holds generically then it holds everywhere.

Let $G$ satisfy the equation $(*)$ generically and let $U$ be a Sylow $^{\circ} 2$-subgroup of $G$. We break the proof into four steps. We will be arguing inductively.

(1) $U$ is 2-unipotent.

(2) If $U$ is nontrivial then $G$ is not simple.

(3) $U$ is normal in $G$.

(4) $G=U \cdot C_{G}(U)$.

For the first point, one may use the theory of [Ch05], already cited above. If $G$ contains a nontrivial decent torus in the sense of that reference, and if $T$ is a maximal such, then $H=N^{\circ}(T)$ is almost disjoint from its conjugates, that is, $H \backslash \bigcup_{g \notin N(H)} H^{g}$ is nongeneric in $H$, and $\bigcup H^{G}$ is generic in $G$. It follows easily, under our present hypotheses, that $H$ also satisfies the equation $(*)$ generically. But $H=C^{\circ}(T)$ as well, and some coset of $T$ in $H$ must satisfy $x^{n}=1$ generically, which is impossible. So there is no nontrivial $p$-torus in $G$ for any $p$. In particular, taking $p=2$, we find that $U$ is 2 -unipotent.

Now if $G$ is simple and $U$ is nontrivial, then $G$ is isomorphic to an algebraic group, by the classification theorem for groups of even type $\mathrm{ABC}$. But then $G$ contains a nontrivial $p$-torus for almost all $p$, a contradiction. The same argument shows that any definable simple section of $G$ must be of degenerate type.

Now we show that $U$ is normal in $G$.

Using some general structure theory following from the classification of the simple groups of even type, one may argue as follows. If $U$ is not normal in $G$, then the subgroup $B(G)$ generated by all the unipotent 2-subgroups of $G$ is nonsolvable, and the nonabelian factors of its socle are algebraic groups of even type; but as we saw above, they must be of degenerate type. We may also argue in a more ad hoc manner as follows.

We may suppose $U \neq 1$. As $G$ is not simple, it is not definably simple, by a result of Zilber. It follows easily that $G$ contains a nontrivial definable connected proper normal subgroup, as otherwise consideration of $G / Z(G)$ (with $Z(G)$ finite) gives a contradiction. Let $K$ be a minimal nontrivial definable connected normal subgroup of $G$. Then $K$ is either abelian, or simple, and in the latter case is of degenerate type. In the abelian case, either $K \leq U$ or $K$ is of degenerate type. The group $U$ acts on $K$, and if $K$ is of degenerate type this action is trivial [Alt, Proposition 2.8.6], [AC03, Proposition 3.2].

We may suppose inductively that $U K / K$ is normal in $G / K$ and thus that $U K$ is normal in $G$. Either $K \leq U$, or $K$ is of degenerate type, in which case $U K$ 
splits as $U \times K$ since $U$ centralizes $K$. In either case it follows at once that $U$ is normal in $G$.

Finally, take a maximal connected definable $G$-invariant series

$$
1=U_{0}<U_{1}<\cdots<U_{n}=U
$$

for $U$ under the action of $G$. As $U$ acts trivially on each successive factor $V_{i}=$ $U_{i} / U_{i-1}, \bar{G}=G / U$ acts on each factor as a group of degenerate type. By $\mathrm{AC} 04$, Lemma 3.13], the Borel subgroups of $\bar{G} / C_{\bar{G}} V_{i}$ are good tori, hence trivial by our current assumptions. It follows that $G$ acts trivially on each such factor, and thus every definable $2^{\perp}$-subgroup of $G$ (including the finite ones) acts trivially on $U$. But $G$ is generated by $U$ and its $2^{\perp}$-subgroups, as it suffices to look at $d(g)$ as $g$ runs over $G$, and thus $G=U \cdot C_{G}(U)$, as claimed.

With this analysis in hand, we may consider the quotient $G / U$, which again satisfies (*) generically. As $G / U$ is of degenerate type it contains no involutions, and thus we may replace $n$ by its odd part. Note that the precise order of the generic element may change in passing from $G$ to $G / U$.

\section{The case of odd primes}

We now prove Theorem 3 , This reads as follows.

Theorem 3. Let $G$ be a connected group of finite Morley rank whose Sylow psubgroups are finite. Then $G$ contains no elements of order $p$.

Here, as it happens, the two possible notions of Sylow $p$-group (maximal $p$ group or maximal locally nilpotent $p$-group) give the statement in question two possible meanings, and we prove this actually in the stronger of the two possible forms, which may be put as follows: a connected $p$-degenerate group of finite Morley rank has no $p$-torsion.

Proof of Theorem 3. Suppose toward a contradiction that $G$ is a $p$-degenerate group of finite Morley rank with nontrivial $p$-torsion. Applying Lemma 3.2 we may suppose that $G$ is simple and that no proper definable connected subgroup of $G$ contains nontrivial $p$-torsion.

By Theorem 1, the prime $p$ is odd.

Now Lemma 4.4 implies that all elements of order $p$ are conjugate in $G$, and in particular each such element is conjugate to its inverse. Hence $G$ contains involutions, and is not of degenerate type.

If $G$ contains a nontrivial unipotent 2-subgroup, then by the results of [ABC], $G$ is a simple algebraic group over an algebraically closed field of characteristic two. But then $G$ contains unbounded $p$-torsion for all odd primes $p$.

It follows that the Sylow ${ }^{\circ} 2$-subgroup of $G$ is a nontrivial 2-torus. In particular $G$ contains nontrivial "decent tori" in the sense of [Ch05], and by the analysis given at the end of that reference, if $T$ is a maximal decent torus (that is, maximal among definable abelian subgroups of the form $d\left(T_{0}\right)$ with $T_{0}$ a divisible torsion subgroup), then $\bigcup\left(N^{\circ}(T)\right)^{G}$ is generic in $G$. 
But by Lemma 4.3, the generic element of $G$ lies outside every proper definable connected subgroup of $G$, and we have a contradiction.

As an application, we can prove the Genericity Conjecture 1 for minimal connected simple groups.

Proposition 8.1. Let $G$ be a minimal connected simple group of finite Morley rank. Then the set of elements of $G$ which belong to some connected nilpotent subgroup of $G$ contains a definable generic subset of $G$.

Proof. Assume the contrary. Then the group contains no decent torus, in view of the results of [Ch05 and a result of Frécon [CJ04, 3.5]. So $G$ is of degenerate type (making use of $\mathrm{ABC}$ to eliminate even and mixed type). On the other hand, there must be $p$-torsion for some prime $p$, so $G$ is not $p$-degenerate. Hence there is an infinite abelian $p$-subgroup of $G$, and as there is no decent torus there is a nontrivial $p$-unipotent subgroup in $G$.

Let $B$ be a Borel subgroup of $G$ with $U_{p}(B)$ nontrivial. As $B$ contains no decent torus, $B / U_{p}(B)$ contains no $p$-torsion, and $B=U_{p}(B) C_{B}\left(U_{p}(B)\right)$.

A generic element $g$ of $B$ has the property that $d(g)$ meets $U_{p}(B)$; otherwise, we would have a generic subset $X$ of $B$ such that for $g \in X$ the group $d(g)$ is $p$-torsion free, and then multiplying $X$ by a nontrivial element of $Z\left(U_{p}(B)\right)$ yields a contradiction.

It follows that $B$ is generically disjoint from its conjugates as otherwise we would have distinct Borel subgroups meeting $U_{p}(B)$ nontrivially, contradicting Bu07, 2.1].

So a generic element of $G$ is conjugate to a generic element of $B$, which lies in a Carter subgroup of $B$ by a result of Frécon [CJ04, 3.5].

Theorem 4, Let $G$ be a connected group of finite Morley rank and $a \in G$ a $p$ element. Then $C^{\circ}(a)$ contains an infinite abelian p-subgroup.

Proof. We follow closely the proof of Theorem 2, analyzing a minimal counterexample.

First one argues that $\bigcup\left[a C^{\circ}(a)\right]^{G}$ is generic in $G$. This proceeds as before. One concludes that the generic element of $G$ lies outside every definable proper connected subgroup.

We claim that $G$ contains no connected normal abelian definable subgroup $A$. Supposing the contrary, by induction there is an infinite connected abelian $p$-group $\bar{P}$ contained in $C_{G / A}(a)$, with preimage $P$ in $G$.

In particular $d(P)\langle a\rangle$ is solvable. By the Hall theory for solvable groups of finite Morley rank, a normalizes a Sylow ${ }^{\circ} p$-subgroup $Q$ of $d(P)$, and $Q$ covers $\bar{P}$. But then $[a, Q] \leq Q \cap A$ and as $Q>Q \cap A$ we find that $C_{Q}(a)$ is infinite and reach a contradiction.

So $G$ contains no connected normal abelian definable subgroup and in particular $Z(G)$ is finite. It now follows that there are no nontrivial $\ell$-tori in $G$ for any $\ell$ : if $T$ is a nontrivial $\ell$-torus then the generic element lies in a conjugate of $C^{\circ}(T)$, and as $T$ cannot be central in $G$ this is a contradiction. 
Now $\bigcup\left[a^{-1} C^{\circ}(a)\right]^{G}$ is also generic in $G$, and it follows easily as in the proof of Lemma 4.4 that $a$ and $a^{-1}$ are conjugate in $G$. This implies that $G$ contains an involution. As $G$ contains no nontrivial 2-torus, it is of even type.

But then the group $B(G)$ generated by the unipotent 2-subgroups of $G$ is a $K$-group by $\mathrm{AC03}$, Proposition 3.4] and the classification of simple groups of even type, and thus has no definable connected simple sections of degenerate type. As there are no definable simple sections of even type either, the group $B(G)$ must be a solvable connected normal subgroup of $G$, and $G$ contains an abelian connected normal subgroup, a contradiction.

\section{Generation}

We turn to Theorem 5, or rather the following, which is marginally stronger in view of Theorem 1 and Lemma 9.2 below.

Proposition 9.1. Let $H$ be a group of finite Morley rank without involutions, and $V$ a 4-group acting definably on $H$. Then

$$
H=\left\langle C(v): v \in V^{\#}\right\rangle .
$$

Proof. Work in $G=H \cdot V$, with $V=\langle i, j\rangle$.

Fix $h \in H$. Then $i$ and $j^{h}$ are not conjugate in $G$, so there is some $u \in I(G)$ commuting with both (indeed, $u \in d\left(i, j^{h}\right)$ ).

As $V$ is a Sylow 2-subgroup of $C(i)$ and $u \in C(i)$, there is some $h_{0} \in C(i)$ so that $u^{h_{0}} \in V$. Similarly there is $h_{1} \in C\left(u^{h_{0}}\right)$ so that $\left(j^{h h_{0}}\right)^{h_{1}} \in V$. Then $j^{h h_{0} h_{1}}=j$ and $h h_{0} h_{1} \in C(j)$, so $h \in C(j) C\left(u^{h_{0}}\right) C(i)$. This proves the claim.

Now in order to recover the statement of Theorem 5, we apply Theorem 1 and also the following general lemma.

Lemma 9.2. Let $G$ be a connected group of finite Morley rank without involutions, and $i$ an involutory automorphism of $G$. Then $C_{G}(i)$ is connected.

Proof. We use a "black box" argument in the style of $\S 5$.

We consider the group $\hat{G}=G \rtimes\langle i\rangle$. For any pair of involutions $i, j \in \hat{G}$ we have $i j \in G$ and thus $d(i j)$ is 2 -torsion-free. It follows that the map

$$
\zeta_{1}: \hat{G} \rightarrow C_{\hat{G}}(i)
$$

introduced in $\S 5$ is well-defined. Observe that the restriction of $\zeta_{1}$ to $G$ carries $G$ into $C_{G}(i)$, and is $C_{G}(i)$-covariant. Thus we find as usual $\operatorname{deg}\left(C_{G}(i)\right) \leq \operatorname{deg}(G)=1$ and $C_{G}(i)$ is connected.

We also give a "lifting" lemma, in the spirit of extending as much as possible of the solvable theory to the general case.

Lemma 9.3. Let $G$ be a group of finite Morley rank and $i$ an involution acting on $G$. Let $H \triangleleft G$ be definable and $i$-invariant, without involutions, and with $i$ acting trivially on $G / H$. Then $G=H \cdot C(i)$. 
Proof. Let $g \in G$, and set $h=[i, g] \in H$. Then $i$ inverts $h$.

As $H$ contains no involutions, the group $d(h)$ is 2-divisible. Take $h_{1} \in d(h)$ with $h_{1}^{2}=h$. Then $i$ inverts $h_{1}$ and hence

$$
\left[i, h_{1}\right]=h_{1}^{2}=[i, g] .
$$

Thus $g h_{1}^{-1} \in C(i)$, and $g \in C(i) H=H C(i)$.

\section{Afterword: Black box groups}

The methods used for the proof of the main result are relatively self-contained. One of the main ingredients comes from an unusual source: "black box group theory" [KS00, via a line of thought represented by [Br00, PW06, AlBo01, Bo02. The subject as a whole deals with the problem of computing in large finite groups which are given in such a form that one can extract elements at random, and perform limited operations or tests on these elements. Among the problems in this area are the determination as to whether the group in question is simple, and its identification if it is. The issue of identification of black box groups is a subject which has remarkable affinities with the subject of groups of finite Morley rank, which can be traced back to a preoccupation with "generic" elements. In the probabilistic setting, this refers to the kinds of elements that appear with probability 1 , while in the model-theoretic setting this refers to the sets of elements which have maximal dimension (Morley rank). Of course, here one may only consider sets which are either measurable or definable, respectively. In black box group theory one can fall back on the classification of the finite simple groups [GLS], whereas in groups of finite Morley rank this is the problem which is under investigation. However, the analogy can be maintained, because with or without a classification theorem, the problem is one of recognition of the specific group with which one is presented, by methods allowed by the corresponding framework. The difference is in outcome: black box group theory delivers practical algorithms (implemented, for example, in GAP) while the theory of groups of finite Morley rank is a conventional mathematical theory dealing in theorems.

In either case, at a certain point, just as in the case of conventional group theory, one requires information about centralizers of involutions. Heretofore in dealing with groups of finite Morley rank we have followed the lead of the finite group theorists, who have a powerful and elegant range of techniques for dealing with this problem. But there is another very direct way to gain a measure of control over the centralizer of an involution which appears to have surfaced first within black box group theory. It is based on elementary properties of dihedral groups which are essential to classical fusion analysis, but via a certain (partial) function $\zeta$ from the group $G$ to the centralizer of an involution which appears to be entirely useless in conventional group theory, probably because it is a partial function, and comes into its own only when it is generically defined (that is, its domain is a generic subset of $G$ ). This is the function $\zeta_{1}$ which we encountered in $\S 5$, along with its companion $\zeta_{0}$. 
From a technical point of view, the virtue of the function $\zeta$ is that it preserves uniform distribution in the probabilistic setting and connectedness in the finite Morley rank setting. Accordingly, if the function $\zeta$ is generically defined, then in the black box setting one can deduce that the centralizer of an involution is again a black box group, and in the finite Morley rank setting one can deduce that the centralizer of an involution is connected if the original group was. We insist here on these parallels because they appear to go more than skin deep.

We mention for the sake of finite group theorists that this technique produces a version of the celebrated $Z^{*}$-theorem [Gl66], proved by methods that have no known analog in the finite case. Indeed, our version assumes connectedness of the ambient group.

Theorem $6\left(Z^{*}\right)$. Let $G$ be a connected group of finite Morley rank, $S$ a Sylow 2-subgroup in $G$ and $i \in S$ an involution. Then either

(a) $i$ is conjugate in $G$ to another involution in $S$, or

(b) $C_{G}(i)$ is connected.

Proof. Let $\mathcal{C}$ be the conjugacy class $i^{G}$. Suppose condition (a) fails: $\mathcal{C} \cap S=\{i\}$. We may suppose $i \notin Z(G)$.

If $j \in \mathcal{C}$ commutes with $i$, then $j=i$. Otherwise, the group $\langle i, j\rangle$ could be conjugated into $S$, giving two conjugates of $i$ in $S$, at least one of which is not $i$.

Fix an involution $j \in \mathcal{C}$.

If $d(i j)$ contains an element $t$ of order 4 , then $i$ inverts $t$ and the subgroup $\langle i, t\rangle$ can be conjugated into $S$. As $\mathcal{C} \cap S=\{i\}$ it follows that $i$ inverts an element $t^{\prime} \in S$ of order 4 , and then $i^{t^{\prime}} \neq i$, contradicting our assumptions.

So $d(i j)$ contains no element of order 4 . Now suppose $d(i j)$ contains an involution $k$. Then (much as in the finite case) $j$ is conjugate to $i k$, and hence $i$ is conjugate to $i k$, which commutes with $i$, giving a contradiction.

So $d(i j)$ contains no involutions. Now holding $i$ fixed, we need to vary $j$ and to consider $d(i j)$ as a function of the element $j$, and we need a generically definable function here. Let $j$ be generic in $\mathcal{C}$ and independent of $i$, and let $\phi(x, y)$ be a formula such that $\phi(x, i j)$ defines $d(i j)$. The set of involutions $j^{\prime} \in \mathcal{C}$ such that $\phi\left(x, i j^{\prime}\right)$ defines an abelian group containing $i j^{\prime}$, inverted by $i$, and without involutions, is a generic subset of $\mathcal{C}$. So letting $\hat{d}\left(i j^{\prime}\right)$ be the group defined by $\phi\left(x, i j^{\prime}\right)$ for such $j^{\prime}$, we can use $\hat{d}$ as a definable approximation to $d$ and define a covariant function $\zeta_{1}: G \rightarrow C(i)$ as we did earlier, then deduce from the connectedness of $G$ that $C(i)$ is connected.

The methods coming from black box group theory offer the outstanding advantage that they do not rely much on induction. In this they resemble the transfer method, which however does not have a direct analog in our context. As degenerate type groups are very poorly understood, this is an essential point. We do in fact use an inductive argument, but only with respect to the presence of involutions; we do not assume anything else about the simple sections which may be present in the group. 
The arguments given here emerged gradually, and have been considerably simplified over time. Earlier arguments used the Alperin-Goldschmidt Theorem Co01 and some of the 0-unipotence theory developed by the second author [Bu04, Bu06]. As these give only special cases of the results given here, we will not elaborate on this point, but as there is a good deal more to be done in the study of groups of degenerate type, these alternative techniques may yet have a role to play.

\section{Acknowledgements}

J. Burdges was supported by NSF Graduate Research Fellowship, and DFG at Würzburg and Bielefeld, grant Te 242/3-1, and acknowledges the hospitality of the Universities of Birmingham and Manchester. G. Cherlin was supported by NSF Grant DMS-0100794.

All authors thank the Newton Institute, Cambridge, for its hospitality during the Model Theory and Algebra program, where the bulk of this work was carried out, as well as CIRM for its hospitality at the September 2004 meeting on Groups, Geometry and Logic, where the seed was planted. Thanks to Altinel for continued discussions all along the way.

\section{References}

[Alt] T. Altınel. Groupes de rang de Morley fini de type pair avec des sous-groupes faiblement inclus. Thèse d'habilitation, Université Claude Bernard, Lyon 1, 2001.

[ABC] T. Altınel, A. V. Borovik, and G. Cherlin. Simple Groups of Finite Morley Rank. Math. Surveys, Amer. Math. Soc. (expected 2008).

[AC03] T. Altınel and G. Cherlin. On groups of finite Morley rank of even type. J. Algebra 264 (2003), 155-185.

[AC04] T. Altınel and G. Cherlin. Simple $L^{*}$-groups of even type with strongly embedded subgroups. J. Algebra 272 (2004), 95-127.

[AlBo01] C. Altseimer and A. Borovik, Probabilistic recognition of orthogonal and symplectic groups. In: Groups and Computation III, W. Kantor and A. Seress (eds.), de Gruyter, Berlin, 2001, 1-20; Corrections (and GAP code): math.GR/0110234.

[Ba96] A. Baudisch. A new uncountably categorical group. Trans. Amer. Math. Soc. 348 (1996), 3889-3940.

[Bo02] A. Borovik. Centralisers of involutions in black box groups. In: Computational and Statistical Group Theory, Contemp. Math. 298, Amer. Math. Soc., 2002, $7-20$.

[BN94] A. Borovik and A. Nesin. Groups of Finite Morley Rank. Clarendon Press, New York, 1994.

[Bo98] E. Bouscaren (ed.). Model Theory and Algebraic Geometry. An Introduction to E. Hrushovski's Proof of the Geometric Mordell-Lang Conjecture. Lecture Notes in Math. 1696, Springer, Berlin, 1998. 
[Br00] J. N. Bray. An improved method for generating the centralizer of an involution. Arch. Math. (Basel) $\mathbf{7 4}$ (2000), 241-245.

[Bu04] J. Burdges. A signalizer functor theorem for groups of finite Morley rank. J. Algebra 274 (2004), 216-229.

[Bu06] J. Burdges. Sylow theory for $p=0$ in groups of finite Morley rank. J. Group Theory 9 (2006), 467-481.

[Bu07] J. Burdges. The Bender method in groups of finite Morley rank. J. Algebra, to appear.

[Ch05] G. Cherlin. Good tori in groups of finite Morley rank. J. Group Theory 8 (2005), 613-621.

[CJ04] G. Cherlin and E. Jaligot. Tame minimal simple groups of finite Morley rank. J. Algebra 275 (2004), 13-79.

[Co01] L.-J. Corredor. Fusion of 2-elements in groups of finite Morley rank. J. Symbolic Logic 66 (2001), 722-730.

[Gl66] G. Glauberman. Central elements in core-free groups. J. Algebra 4 (1966), 403-420.

[GLS] D. Gorenstein, R. Lyons, and R. Solomon. The Classification of the Finite Simple Groups. Math. Surveys Monogr. 40.1-6, Amer. Math. Soc., 1994-2005.

[HZ96] E. Hrushovski and B. Zilber. Zariski geometries. J. Amer. Math. Soc. 9 (1996), $1-56$.

[Ja99] Kh. Jaber. Équations génériques dans un groupe stable nilpotent. J. Symbolic Logic 64 (1999), 761-768.

[Ja06] E. Jaligot. Generix never gives up. J. Symbolic Logic 71 (2006), 599-610.

[KS00] W. M. Kantor and A. Seress. Black box classical groups. Mem. Amer. Math. Soc. 149 (2000), no. 708.

[PW06] C. W. Parker and R. A. Wilson. Recognizing simplicity of black-box groups. Preprint, 2006.

[Po90] B. Poizat. Équations génériques. In: Proceedings of the 8th Easter Conference on Model Theory (Wendisch-Rietz, 1990), Seminarber. 110, Humboldt Univ., Berlin, 1990, 131-138.

[Po01] B. Poizat. Groupes Stables. Nur al-Mantiq wal-Ma'rifah, Villeurbanne, France, 1987; English transl., Amer. Math. Soc., 2001.

[Sc01] T. Scanlon. Diophantine geometry from model theory. Bull. Symbolic Logic 7 (2001), 37-57.

\author{
Alexandre Borovik \\ School of Mathematics \\ University of Manchester \\ Oxford Road, Manchester M13 9PL \\ U.K. \\ e-mail: borovik@manchester.ac.uk
}


Jeffrey Burdges

Université de Lyon

Université Lyon 1

CNRS, UMR 5208 Institut Camille Jordan

43, blvd du 11 novembre 1918

F-69622 Villeurbanne Cedex, France

e-mail: burdges@math.rutgers . edu

Gregory Cherlin

Department of Mathematics

Rutgers University

110 Frelinghuysen Rd.

Piscataway, NJ 08854, U.S.A.

To access this journal online: www.birkhauser.ch/sm 